\title{
The Principle of Indifference Does Not Lead to Contradictions
}

\author{
Wolfgang Tschirk ${ }^{1}$ \\ ${ }^{1}$ mathecampus, Vienna, Austria \\ Correspondence: Wolfgang Tschirk, mathecampus, Mariahilfer Straße 136, 1150 Wien, Austria. Tel: 43-680-126-8416. \\ E-mail: wolfgang.tschirk@mathecampus.at
}

Received: May 6, 2016 Accepted: May 20, 2016 Online Published: June 22, 2016

doi:10.5539/ijsp.v5n4p79 URL: http://dx.doi.org/10.5539/ijsp.v5n4p79

\begin{abstract}
The Principle of Indifference says that if there are a finite number of propositions and a state of knowledge according to which none of the propositions is more plausible than any other, then, conditional on that knowledge, all of them have the same probability. Most researchers reject the principle because there exist counterexamples believed to prove that it leads to contradictions. We analyse three examples representative of the objections to the principle and show that, rather than disproving it, they suffer from a common error in applying it. From this and the fact that the Principle of Indifference complies with plausible reasoning we conclude that it does not lead to contradictions.
\end{abstract}

Keywords: Principle of Indifference, Principle of Insufficient Reason

\section{Introduction}

The Principle of Indifference, which dates back to Jakob Bernoulli, can be expressed as follows:

If, given some proposition $C$, one of the propositions $A_{1}, \cdots, A_{n}$ must be true and the others must be false and none of the $A_{i}$ is more plausible than any other, then, conditional on $C$, all $A_{i}$ have the same probability $p\left(A_{i} \mid C\right)=1 / n$.

$C$ is often referred to as someone's (prior) knowledge, which may or may not contain reasons to favour some proposition over some other. The rule became known as the Principle of Insufficient Reason. In 1921, Keynes introduced the term Principle of Indifference (Keynes, 1921). Keynes raised doubt whether the principle was valid and presented counterexamples intended to prove that it leads to contradictions and is therefore invalid. Other authors have commented on these examples and added their own.

Due to the presumed contradictions, most researchers have rejected and still reject the principle (Bartelborth, 2012; Carnap, 1966; Howie, 2002; Robert, 2007; van Fraassen, 1989). However, in the 1950s, Jaynes derived it from a set of desiderata for plausible reasoning (Jaynes 1958, 2003). In his probability theory, the principle is a proven theorem; it cannot be contradictory there, unless the whole theory were contradictory. If it is not, it must be possible to eliminate the contradictions on the ground of Jaynes' desiderata. This is what we are going to undertake.

\section{Counterexamples to the Principle}

We start with three examples Keynes gave in order to demonstrate where the Principle of Indifference fails. For ease of presentation, we adapt the wording without substantially altering the content.

1. The colour of the book: A person who knows nothing about the colour of a certain book must, according to the Principle of Indifference, assign the proposition $R:=$ The book is red the same probability as its contrary $\bar{R}=$ The book is not red, namely $p(R)=1 / 2$. For the same reason the person must assign the proposition $B:=$ The book is blue the probability $p(B)=1 / 2$ and the proposition $G:=$ The book is green the probability $p(G)=1 / 2$. But now the proposition $R \vee B \vee G=$ The book is red or blue or green would have the probability $p(R \vee B \vee G)=$ $p(R)+p(B)+p(G)=3 / 2$, which is impossible as probabilities do not exceed unity.

2. Sizes of countries: A person who knows nothing about the sizes of countries must, according to the Principle of Indifference, assign the proposition $E:=$ England is bigger than France the same probability as its contrary $\bar{E}=$ England is not bigger than France, namely $p(E)=1 / 2$. For the same reason the person must assign the proposition $B:=$ The British Isles are bigger than France the probability $p(B)=1 / 2$. But this is impossible, since England is only a part of the British Isles; the Isles must surpass France in size with higher probability than England alone. 
3. Specific volume vs. specific density: A person who only knows that the specific volume of some substance lies between 1 and 3 (measured in some unit) must, according to the Principle of Indifference, assign the propositions $V:=$ The specific volume lies between 1 and 2 and its contrary $\bar{V}=$ The specific volume lies between 2 and 3 the probabilities $p(V)=p(\bar{V})=1 / 2$. The reciprocal of the specific volume is the specific density. The person only knows that it lies between $1 / 3$ and 1 , and must therefore assign the proposition $D:=$ The specific density lies between $1 / 3$ and $2 / 3$ the same probability as its contrary $\bar{D}=$ The specific density lies between $2 / 3$ and 1 , namely $p(D)=1 / 2$. But this is impossible, since $D$ says (though in other words) that the specific volume lies between $3 / 2$ and 3 and must be more probable than $\bar{V}$.

As far as we know, every serious objection to the Principle of Indifference is a modification of one of these examples, corresponding to one of the following patterns:

1. The pattern of The colour of the book: If one out of two equally probable propositions (The book is red and The book is not red) is dissected (as The book is not red is dissected into The book is blue, The book is green, and maybe others, too), the resulting ones (The book is red, The book is blue, The book is green, etc.) cannot be equally probable; yet the Principle of Indifference says they are equally probable.

2. The pattern of Sizes of countries: A proposition (England is bigger than France) cannot have the same probability as an obviously more probable one (The British Isles are bigger than France); yet the Principle of Indifference says it has the same probability.

3. The pattern of Specific volume vs. specific density: Uniform distribution on one scale (equal probabilities for equally large intervals on the specific volume scale) leads to non-uniform distribution on a different scale (nonequal probabilities for equally large intervals on the specific density scale); yet the Principle of Indifference requires uniform distributions on both scales.

Among the objections that correspond to these patterns are: the partitioning incoherence of Laplace's equiprobability claimed by Robert (2007), corresponding to pattern 1; Carnap's life on Mars (Carnap, 1966), corresponding to pattern 2; Keynes' urn with black and white balls (Keynes, 1921), van Fraassen's cube factory (van Fraassen, 1989) and von Mises' wine/water-paradox (Mikkelson, 2004), all corresponding to pattern 3

At first sight, the examples seem to disprove the Principle of Indifference; however, we will find that they suffer from a common error in applying it.

\section{Desiderata for Plausible Reasoning}

We base our analysis on Jaynes' desiderata for plausible reasoning, i.e. for assigning degrees of plausibility to propositions (in Jaynes' terminology, such plausibility assignment is called a conclusion):

(I) Degrees of plausibility are represented by real numbers.

(II) Plausible reasoning qualitatively corresponds with common sense.

(IIIa) If a conclusion can be reasoned in more than one way, every way leads to the same result.

(IIIb) Every conclusion is based on all available knowledge.

(IIIc) Equivalent states of knowledge lead to equivalent conclusions.

Desideratum (I), together with the convention that a greater plausibility shall correspond to a greater number, guarantees that 1) any two propositions $A$ and $B$ can be compared with respect to plausibility such that either $A$ is more plausible than $B$, or $B$ is more plausible than $A$, or $A$ and $B$ are equally plausible, and 2) if $A$ is more plausible than $B$, and $B$ is more plausible than $C$, then $A$ is more plausible than $C$.

From desideratum (II), the following rule, which we call implication rule, can be derived:

If, given $C, A$ implies $B$ and $B$ does not imply $A$, then, given $C, B$ is more plausible than $A$.

It corresponds with common sense because, given $C, B$ is true whenever $A$ is true, but $B$ can even be true when $A$ is false. Desiderata (IIIa)-(IIIc) ensure that reasoning is consistent. 


\section{Analysis of the Counterexamples}

\subsection{Example 1: The colour of the book}

We start the examination by asking for the knowledge based on which the probabilities are assigned. Let $C_{R}$ be some knowledge according to which $R$ is equally plausible as $\bar{R}$; then, following the Principle of Indifference, the probability of $R$, given $C_{R}$, is $1 / 2: p\left(R \mid C_{R}\right)=1 / 2$. Let $C_{B}$ be some knowledge which makes $B$ equally plausible as $\bar{B}$, and $C_{G}$ some knowledge which makes $G$ equally plausible as $\bar{G}$, then $p\left(B \mid C_{B}\right)=1 / 2$ and $p\left(G \mid C_{G}\right)=1 / 2$. Now each of the colours has a probability of $1 / 2$ to be the colour of the book, but these probabilities are conditional on different knowledge; and

$$
p\left(R \mid C_{R}\right)+p\left(B \mid C_{B}\right)+p\left(G \mid C_{G}\right)=3 / 2
$$

does not violate any rule of probability (as $C_{R}, C_{B}$ and $C_{G}$ are different, the sum on the left side of the equation is not a probability). This has already been observed by Jeffreys in his review of Keynes' work (Jeffreys, 1922).

As long as $C_{R}, C_{B}$ and $C_{G}$ are not identical, Example 1 does not disprove the Principle of Indifference. The principle would fail only if there existed a state of knowledge which made $R$ equally plausible as $\bar{R}$ and, at the same time, $B$ equally plausible as $\bar{B}$ and $G$ equally plausible as $\bar{G}$. Now we prove by contradiction that such knowledge cannot exist.

Assume that there exists some knowledge $C$ according to which each of the propositions $R, B$ and $G$ is equally plausible as its respective contrary. First we note that $C$ allows red, blue and green to be possible colours of the book (if, for instance, red were impossible, then $R$ would be less plausible than $\bar{R}$ ). Then, given $C, R$ implies $\bar{B}$ but $\bar{B}$ does not imply $R$ (a red book is clearly non-blue, whereas a non-blue book does not have to be red); following the implication rule, $\bar{B}$ is more plausible than $R$, conditional on $C$. Using Jaynes' notation, where $A \mid B$ stands for the plausibility of $A$, given $B$, we have thus found

$$
\bar{B}|C>R| C .
$$

An analogous reasoning shows that $\bar{R}$ is more plausible than $B$, conditional on $C$ :

$$
\bar{R}|C>B| C .
$$

Now remember that $C$ is assumed to make $R$ and $\bar{R}$ equally plausible:

$$
R|C=\bar{R}| C .
$$

Putting these relations together, we arrive at

$$
\bar{B}|C>R| C=\bar{R}|C>B| C,
$$

which contradicts the assumption that $C$ makes $B$ and $\bar{B}$ equally plausible. Therefore, a state of knowledge according to which each of the propositions $R, B$ and $G$ were equally plausible as its respective contrary does not exist.

From the above follows that Example 1 does not disprove the Principle of Indifference; its paradox results from the assumption of prior knowledge which cannot exist.

\subsection{Example 2: Sizes of countries}

As in Example 1, we start by asking for the knowledge based on which the probabilities are assigned. Let $C_{E}$ be some knowledge according to which $E$ is equally plausible as $\bar{E}$; then $p\left(E \mid C_{E}\right)=1 / 2$. Let $C_{B}$ be some knowledge which makes $B$ equally plausible as $\bar{B}$; then $p\left(B \mid C_{B}\right)=1 / 2$. We thus obtain

$$
p\left(E \mid C_{E}\right)=p\left(B \mid C_{B}\right) ;
$$

but although England is a part of the British Isles, this equality is not impossible since the probabilities are conditional on different knowledge.

The Principle of Indifference would fail on Example 2 only if there existed a state of knowledge which contained the information that England is a part of the British Isles and, at the same time, made $E$ equally plausible as $\bar{E}$ and $B$ equally plausible as $\bar{B}$. We prove by contradiction that such knowledge cannot exist.

Assume that there exists some knowledge $C$ according to which England is a part of the British Isles and each of the propositions $E$ and $B$ is equally plausible as its respective contrary. Then, given $C, E$ implies $B$ but $B$ does not imply $E$ (if England is bigger than France, then the British Isles also are; however, from the premise that the British Isles are bigger than France, one cannot conclude that England also is); following the implication rule, $B$ is more plausible than $E$, conditional on $C$ :

$$
B|C>E| C .
$$


On the other hand, given $C, \bar{B}$ implies $\bar{E}$ but $\bar{E}$ does not imply $\bar{B}$ (if the British Isles are not bigger than France, then England is also not; however, from the premise that England is not bigger than France, one cannot conclude that the British Isles are not); following the implication rule, $\bar{E}$ is more plausible than $\bar{B}$, conditional on $C$ :

$$
\bar{E}|C>\bar{B}| C .
$$

Now remember that $C$ is assumed to make $E$ and $\bar{E}$ equally plausible:

$$
E|C=\bar{E}| C .
$$

Putting these relations together, we arrive at

$$
B|C>E| C=\bar{E}|C>\bar{B}| C,
$$

which contradicts the assumption that $C$ makes $B$ and $\bar{B}$ equally plausible. Therefore, a state of knowledge according to which England is a part of the British Isles and each of the propositions $E$ and $B$ were equally plausible as its respective contrary does not exist.

It follows that Example 2 does not disprove the Principle of Indifference; its paradox results from the assumption of prior knowledge which cannot exist.

\subsection{Example 3: Specific volume vs. specific density}

Again we ask for the knowledge based on which the probabilities are assigned. Let $C_{V}$ be some knowledge according to which $V$ is equally plausible as $\bar{V}$; then $p\left(\bar{V} \mid C_{V}\right)=1 / 2$. Let $C_{D}$ be some knowledge which makes $D$ equally plausible as $\bar{D}$; then $p\left(D \mid C_{D}\right)=1 / 2$. We thus obtain

$$
p\left(\bar{V} \mid C_{V}\right)=p\left(D \mid C_{D}\right) ;
$$

but although specific volume and specific density are reciprocals of each other, this equality is not impossible since the probabilities are conditional on different knowledge.

The Principle of Indifference would fail on Example 3 only if there existed a state of knowledge which contained the information that specific volume and specific density are reciprocals of each other and, at the same time, made $V$ equally plausible as $\bar{V}$ and $D$ equally plausible as $\bar{D}$. We prove by contradiction that such knowledge cannot exist.

Assume that there exists some knowledge $C$ according to which specific volume and specific density are reciprocals of each other and each of the propositions $V$ and $D$ is equally plausible as its respective contrary. Then, given $C, \bar{V}$ (The specific volume lies between 2 and 3 ) implies $D$ (equivalent to The specific volume lies between $3 / 2$ and 3 ) but $D$ does not imply $\bar{V}$; following the implication rule, $D$ is more plausible than $\bar{V}$, conditional on $C$ :

$$
D|C>\bar{V}| C .
$$

On the other hand, given $C, \bar{D}$ (The specific density lies between 2/3 and 1 ) implies $V$ (equivalent to The specific density lies between $1 / 2$ and 1 ) but $V$ does not imply $\bar{D}$; following the implication rule, $V$ is more plausible than $\bar{D}$, conditional on $C$ :

$$
V|C>\bar{D}| C .
$$

Now remember that $C$ is assumed to make $V$ and $\bar{V}$ equally plausible:

$$
V|C=\bar{V}| C .
$$

Putting these relations together, we arrive at

$$
D|C>\bar{V}| C=V|C>\bar{D}| C,
$$

which contradicts the assumption that $C$ makes $D$ and $\bar{D}$ equally plausible. Therefore, a state of knowledge according to which specific volume and specific density are reciprocals of each other and each of the propositions $V$ and $D$ were equally plausible as its respective contrary does not exist.

It follows that Example 3 does not disprove the Principle of Indifference; its paradox results from the assumption of prior knowledge which cannot exist.

\section{Results}

We have analysed three examples representative of the objections to the Principle of Indifference and found that, rather than disproving the principle, they suffer from a common error in applying it. The error can be described as follows: First, 
a state of knowledge is assumed which contains no reason to favour any proposition (out of a certain set of propositions) over its contrary; second, based on this knowledge, each proposition is assigned a probability in accordance with the Principle of Indifference; third, the probability assignment is shown to be contradictory and the contradiction is attributed to the Principle of Indifference. However, as we have proven for each example, a state of knowledge which contains no reason to favour any of said propositions over its contrary and, at the same time, renders the resulting probability assignment inconsistent is impossible.

Therefore, none of the examples disproves the Principle of Indifference; the contradiction is always caused by the assumption of prior knowledge which cannot exist.

\section{Discussion and Conclusions}

The Principle of Indifference is a proven theorem in a probability theory that emerged from a set of desiderata for plausible reasoning. On the other hand, there exist counterexamples believed to show that it leads to contradictions. Being proven, the principle cannot be contradictory, unless the whole theory were contradictory. If it is not, it must be possible to eliminate the contradictions on the ground of the desiderata the theory is built upon.

Based on these desiderata, we have analysed three counterexamples and found that in none of them the principle fails. Whenever a contradiction arises, it is caused by the erroneous assumption that there be no reason to favour any relevant proposition over its contrary. In each of the examples we have found such reason; it has never been an empirical reason, it was always a logical one: Whatever prior knowledge one has with respect to the colour of a certain book and whether or not it contains reasons to favour or disfavour red, to favour or disfavour blue or to favour or disfavour green - it is logically impossible that each of the propositions The book is red, The book is blue and The book is green be exactly as plausible as its negation; and analogous arguments hold with respect to the other examples.

Some opponents to the Principle of Indifference consider only empirical knowledge as relevant knowledge. In his Sizes of countries example, Keynes explicitely rejects a way out of the contradiction based on logic. However, the use of logical evidence in plausibility assignments is mandatory because of two reasons: First, it is required by desideratum (IIIb); second, consistent reasoning would be impossible if the rules of logic could be arbitrarily ignored, in particular, fulfillment of the desiderata (IIIa) and (IIIc) would not be guaranteed.

As far as we know, every serious objection to the Principle of Indifference corresponds to the pattern of one of the examples we have analysed. If this is true, then the principle does not lead to contradictions at all.

\section{References}

Bartelborth, T. (2012). Die erkenntnistheoretischen Grundlagen induktiven Schließens.

Ebook, URN=http://nbn-resolving.de/urn:nbn:de:bsz:15-qucosa-84565

Carnap, R. (1966). Philosophical Foundations of Physics. An Introduction to the Philosophy of Science. New York, NY: Basic Books.

Howie, D. (2002). Interpreting Probability. Controversies and Developments in the Early Twentieth Century. New York, NY: Cambridge University Press.

Jaynes, E. T. (1958). Probability Theory in Science and Engineering. Dallas, TX: Socony Mobile Oil Company.

Jaynes, E. T. (2003). Probability Theory. The Logic of Science. New York, NY: Cambridge University Press.

Jeffreys, H. (1922). The Theory of Probability. Nature 109, 132-133. http://dx.doi.org/10.1038/109132a0

Keynes, J. M. (1921). A Treatise on Probability. London, England: MacMillan \& Co.

Mikkelson, J. M. (2004). Dissolving the Wine/Water Paradox. British Journal for the Philosophy of Science 55, $137-145$. http://dx.doi.org/10.1093/bjps/55.1.137

Robert, C. P. (2007). The Bayesian Choice. From Decision-Theoretic Foundations to Computational Implementation. New York, NY: Springer.

Van Fraassen, B. C. (1989). Laws and Symmetry. New York, NY: Oxford University Press.

\section{Copyrights}

Copyright for this article is retained by the author(s), with first publication rights granted to the journal.

This is an open-access article distributed under the terms and conditions of the Creative Commons Attribution license (http://creativecommons.org/licenses/by/3.0/). 